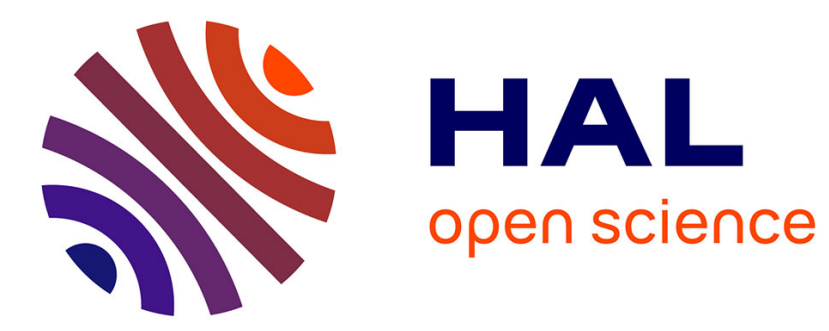

\title{
Continuous Binder-Free Fibers of Pure Imogolite Nanotubes
}

\author{
Joseph F Moore, Erwan Paineau, Pascale Launois, Milo S P Shaffer
}

\section{To cite this version:}

Joseph F Moore, Erwan Paineau, Pascale Launois, Milo S P Shaffer. Continuous Binder-Free Fibers of Pure Imogolite Nanotubes. ACS Applied Materials \& Interfaces, 2021, 13 (15), pp.17940-17947. 10.1021/acsami.1c00971 . hal-03278907

\section{HAL Id: hal-03278907 https://hal.science/hal-03278907}

Submitted on 13 Jul 2021

HAL is a multi-disciplinary open access archive for the deposit and dissemination of scientific research documents, whether they are published or not. The documents may come from teaching and research institutions in France or abroad, or from public or private research centers.
L'archive ouverte pluridisciplinaire HAL, est destinée au dépôt et à la diffusion de documents scientifiques de niveau recherche, publiés ou non, émanant des établissements d'enseignement et de recherche français ou étrangers, des laboratoires publics ou privés. 


\section{Continuous Binder-Free Fibers of Pure Imogolite}

\section{Nanotubes}

Joseph F. Moore, ${ }^{1}$ Erwan Paineau, ${ }^{2}$ Pascale Launois, ${ }^{2, *}$ Milo S. P. Shaffer ${ }^{1,3, *}$

${ }^{1}$ Department of Materials, Imperial College London, Exhibition Road, London, SW7 2AZ, UK

${ }^{2}$ Université Paris-Saclay, CNRS, Laboratoire de Physique des Solides, 91405 Orsay, France

${ }^{3}$ Department of Chemistry, Imperial College London, Exhibition Road, London, SW7 2AZ, UK

Keywords: nanotube, fiber, imogolite, alignment, humidity, jamming, spinning

\section{ABSTRACT}

Imogolite nanotubes display a range of useful properties and provide an ideal material system to study the assembly of nanomaterials into macroscopic fibers. A method of wet spinning pure, binder-free imogolite fibers has been developed using double-walled germanium imogolite nanotubes. Nanotube aspect ratio can be controlled during the initial synthesis and is critical to the spinning process. Fibers made from short nanotubes $(<100 \mathrm{~nm})$ have very low gel-strengths, whilst dopes with longer nanotubes $(500-1000 \mathrm{~nm})$ are readily spinnable. The tensile behaviour of the resulting imogolite nanotube fibers is strongly influenced by relative humidity $(\mathrm{RH})$, with a modulus of $30 \mathrm{GPa}$ at $10 \% \mathrm{RH}$ compared to $2.8 \mathrm{GPa}$ at $85 \% \mathrm{RH}$, as well as a change in failure mode. This result highlights the importance of 
inter-nanotube interactions in such assemblies and provides a useful strategy for further exploration. Interestingly, in the absence of a matrix phase, a degree of misorientation appears to improve load transfer between the individual INTs within the porous fiber, likely due to an increase of the number of inter-particle contacts. Imogolite nanotubes are an appealing analogue to other nanotube fiber systems, and it is hoped that learnings from this system can also be used to improve carbon nanotube fibers.

\section{INTRODUCTION}

Nanotubes and nanorods are promising candidates for producing high performance fibers, due to their excellent intrinsic mechanical properties and compatible 1D morphology. However, the mechanical properties of macroscale fibers are typically significantly lower than those of the constituent nanomaterials, due to a combination of processing challenges and weak inter-molecular interactions. To date, most studies have focused on carbon nanotube $(\mathrm{CNT})$ based fibers, using both dry and wet spinning methods, ${ }^{1-4}$ fiber properties have typically been improved by increasing the aspect ratio and alignment of the nanotubes. CNT processing has proved particularly challenging given their poor solubility in convenient solvents, and tendency to agglomerate. Understanding and optimizing these spinning processes is generally hindered by the difficulty in measuring CNT orientation within the fibers. Strong light absorbance limits the use of optical methods, whilst polydispersity, irregular packing, and weak scattering makes it challenging to study single filament samples with X-rays efficiently. ${ }^{5}$

Imogolite nanotubes (INTs) are an appealing analogue for CNTs due to their monodisperse diameter and spontaneous dispersion in deionized water, which enables simple solution processing. ${ }^{6}$ The archetypal INT is single-walled and comprises a curved outer gibbsite $\mathrm{Al}(\mathrm{OH})_{3}$ sheet inside which isolated $\mathrm{O}_{3} \mathrm{SiOH}$ tetrahedra are located. ${ }^{7,8}$ However, a variety of INTs can be synthesized via sol-gel methods using different precursors, particularly ones 
with Ge substituting for $\mathrm{Si}^{9}{ }^{9}$ in either double-walled or single-walled form. ${ }^{10,}{ }^{11}$ Similarly to solutions of CNT polyelectrolytes, ${ }^{12-14}$ INTs form liquid crystalline mesophases which can be reoriented either by electrical or flow fields, ${ }^{15,16}$ potentially leading to highly aligned fibers. In contrast to CNTs, INT solutions are optically transparent which greatly aids the use of polarized optical microscopy $(\mathrm{POM})$ in their characterization. Additionally, $\mathrm{Al}$ and $\mathrm{Ge}$ atoms have much greater X-ray scattering cross-sections than carbon, which facilitates the use of lab-source X-rays for studying the structure of both dispersions and macroscale fiber assemblies. The hydrophilicity of INTs also enables the tuning of nanotube interactions by varying humidity. INTs have been shown to adsorb up to $80 \%$ of their weight in water at $95 \%$ relative humidity $(\mathrm{RH})^{17}$ with the water adsorbed in the internal cavity, interstitially between the walls of double-walled nanotubes and crucially on the outer surface. ${ }^{18}$ In principle, by varying the humidity, the strength of inter-nanotube hydrogen bonding should be modified, enabling the influence of shear interactions on the mechanical properties of nanotube-based fibers to be studied. ${ }^{19,20}$

In addition to their appeal as a model system, INTs are increasingly of interest in their own right, with potential applications in molecular filtration, catalysis and molecular transport and release. ${ }^{6}$ Whilst INTs have lower absolute mechanical properties than CNTs, the strength and stiffness are still significant, with predicted elastic modulus between 100 and $400 \mathrm{GPa}^{21-24}$ More generally, they have complementary properties to CNTs in electrical conductivity, color and hydrophilicity. Nanocomposite and hybrid films of INTs with polymers and biomaterials have previously been produced ${ }^{25-27}$ and recently continuous INT-polyvinyl alcohol composite fibers have been prepared which show a degree of self-healing at remarkably high absolute strengths. ${ }^{28}$ However, as yet, no macroscale fibers have been reported from pure INTs. This study, therefore, investigates the possibility of using wet 
spinning to create pure, binder-free imogolite nanotube fibers and explores the effects of nanotube length, alignment, and hydration on fiber processability and mechanical properties.

\section{SYNTHESIS OF DW Ge-INTs}

It is challenging to spin fibers from nanomaterials such as CNTs and nanocellulose, if the aspect ratio is too low. ${ }^{29,30}$ Therefore, in order to enhance spinnability in this work, the INT synthesis was developed to provide high aspect ratio feedstocks. Compared to the conventional synthesis route using sodium hydroxide, ${ }^{31}$ the in-situ production of hydroxyl ions by thermal decomposition of urea produces significantly longer double-walled germanium imogolite nanotubes (DW Ge-INTs) after 5 days of reaction; the inner and outer diameters remain consistently $1.6 \mathrm{~nm}$ and $4.3 \mathrm{~nm}$, respectively. ${ }^{32}$ Although several studies have been devoted to the mechanisms of nanotube growth during synthesis, ${ }^{33,34}$ the effect of long reaction times ( $>5$ days) remains largely unexplored. In this work, the ageing time of the nanotube synthesis was varied from 5 to 40 days (feedstocks INT-5d, INT-12d, INT-20d etc.) to create spinning dopes with an expected further increase in length. The length distribution
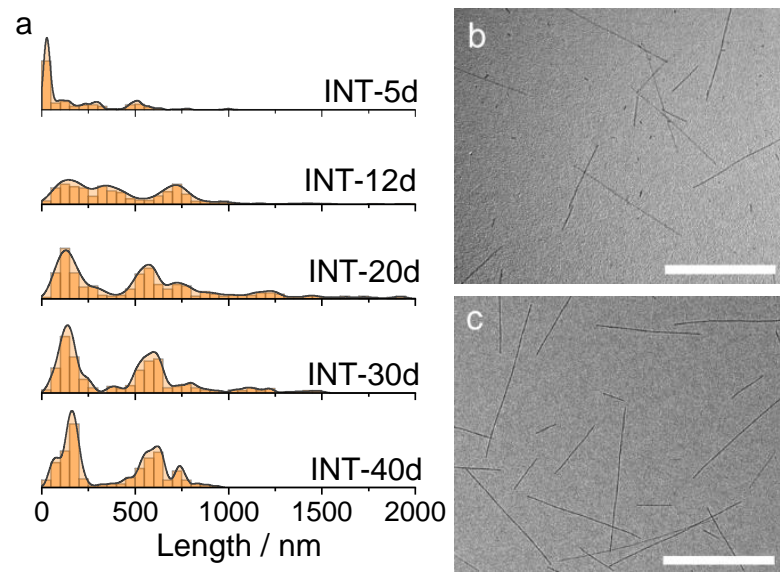

of each INT feedstock was determined by measuring the length of at least 200 nanotubes over several TEM images (Figure 1).

Figure 1.(a) Length number distributions of INT feedstocks determined by measurements in TEM, illustrative distribution curves have been generated with a Gaussian kernel density 
estimator (b,c) Representative TEM images of (b) INT-5d and (c) INT-40d. Scale bars $500 \mathrm{~nm}$.

INT-5d is dominated by short nanotubes $(<100 \mathrm{~nm})$. With further incubation, these nanotubes were consumed to produce a bimodal distribution with average lengths centred at $150 \mathrm{~nm}$ and $600 \mathrm{~nm}$. Beyond 20 days incubation, little further change to the distribution was identified. Oriented attachment (OA) of formed nanotubes has previously been proposed as a key mechanism of INT growth after consumption of the initial precursors and topological rearrangment of protoimogolite nanostructures. ${ }^{33,}{ }^{35}$ However, this growth mechanism is expected to occur extremely slowly at larger nanotube lengths due to the $L^{-4}$ dependency of tip-tip collision frequency, explaining the lack of meaningful further growth after 20 days. ${ }^{36}$ IR spectroscopy indicates no significant difference in the chemical bonding within the INTs for the different reaction periods (Figure S1).

\section{SPINNING DW Ge-INT FIBERS}

Binder-free imogolite fibers were spun by injecting an aqueous spinning dope solution of DW Ge-INTs $(9 \mathrm{~g} / \mathrm{L})$ through a needle into a coagulation bath of calcium chloride in water $(300 \mathrm{~g} / \mathrm{L})$. The spinning dopes exhibited strong birefringence (Figure S2), indicative of an ordered mesophase observed previously in both carbon and imogolite nanotube dispersions, ${ }^{16,37,38}$ which should be ideal for liquid crystalline wet spinning. The high ionic strength of the coagulant $(\mathrm{I}=8.1 \mathrm{M})$ enabled quick gelation of the spinning dope. At slow injection rates, a transparent gel monolith formed at the tip of the needle. Increasing the injection velocity led to the formation of a gel-like proto-fiber that could be manipulated and then collected on a winding wheel to provide draw during the spinning process (Supplementary Movie 1). The fracture strength of the proto-fibers, and thus their ease of manipulation, depended strongly upon the length distribution of the nanotube feedstock. The 
longer nanotube feedstocks (12d-40d) could be easily spun, whereas frequent filament breakage occurred with the INT-5d feedstock. It was observed that the filament breakage consistently occurred at the coagulant/air interface, indicating that the surface tension of the coagulant liquid imparts sufficient force to overcome the gel strength of the fiber. The stress in a cylindrical fiber being withdrawn from a liquid is inversely proportional to its radius (Figure S3). Thus, increasing the radius of the gel fiber should reduce the probability of filament breakage. In accordance with this theory, the INT-5d feedstock was found to be unspinnable using a 24 gauge needle (internal diameter, ID $311 \mu \mathrm{m}$ ) but spinnable using a 21 gauge needle (ID $514 \mu \mathrm{m}$ ). The gel strength of the INT-5d proto-fiber was therefore estimated to be approximately $500 \mathrm{~Pa}$. The other feedstocks, from INT-12d to INT-40d, were successfully drawn from the coagulant without breakage using a 27 gauge needle (ID $210 \mu \mathrm{m}$ ) implying a gel strength of at least $1.5 \mathrm{kPa}$. These gel strengths are relatively low, requiring careful handling of the proto-fibers before collection and drying. Polymer/INT hybrid hydrogels typically have significantly higher fracture strengths; for comparison polyacrylamide hydrogels with a 5\% INT loading have been produced with a fracture strength of $220 \mathrm{kPa}^{39}$ and hyaluronic acid/INT hydrogels with $1 \%$ and $10 \%$ INT loading have shown fracture strengths of $20 \mathrm{kPa}$ and $100 \mathrm{kPa}$ respectively. ${ }^{40}$ Unlike polymers, the rigid rod INT system is held together only by friction and jamming, rather than molecular entanglement.

After washing in deionized water and drying in ambient conditions for 48 hours, the resulting INT fibers were robust and readily handled with an average diameter of $\sim 20 \mu \mathrm{m}$, textured surfaces and irregular cross-sections typical of nanomaterial fibers ${ }^{41}$ (Figure 2a-c and S4). Energy dispersive X-ray spectroscopy indicates that the dry fibers are mostly comprised of aluminium, germanium and oxygen with a small residue of calcium ( 3 at $\%)$ and chlorine $(6$ at $\%)$ remaining after the washing procedure (Figure S4). As expected from the 
Ge-INT structural formula $\left(\mathrm{GeAl}_{2} \mathrm{O}_{7} \mathrm{H}_{4}\right)$, the measured atomic ratio of aluminium to germanium was $2: 1$. Although, it is challenging to image the internal structure by SEM, due to the low electrical conductivity and small INT diameter, the fibre microstructure appears uniform with no evidence of a skin-core texture. The packing density of the fibers can be estimated from the dope concentration, needle area, draw ratio and fiber cross-sectional area and density of a DW Ge-INT (Supplementary Note 1). This estimate yields packing densities around $45-55 \%$, similar to that of floating catalyst chemical vapor deposition CNT fibers $(\sim 35-60 \%),{ }^{42}$ but lower than highly optimized wet spun CNT fibers $(\sim 75 \%){ }^{29}$
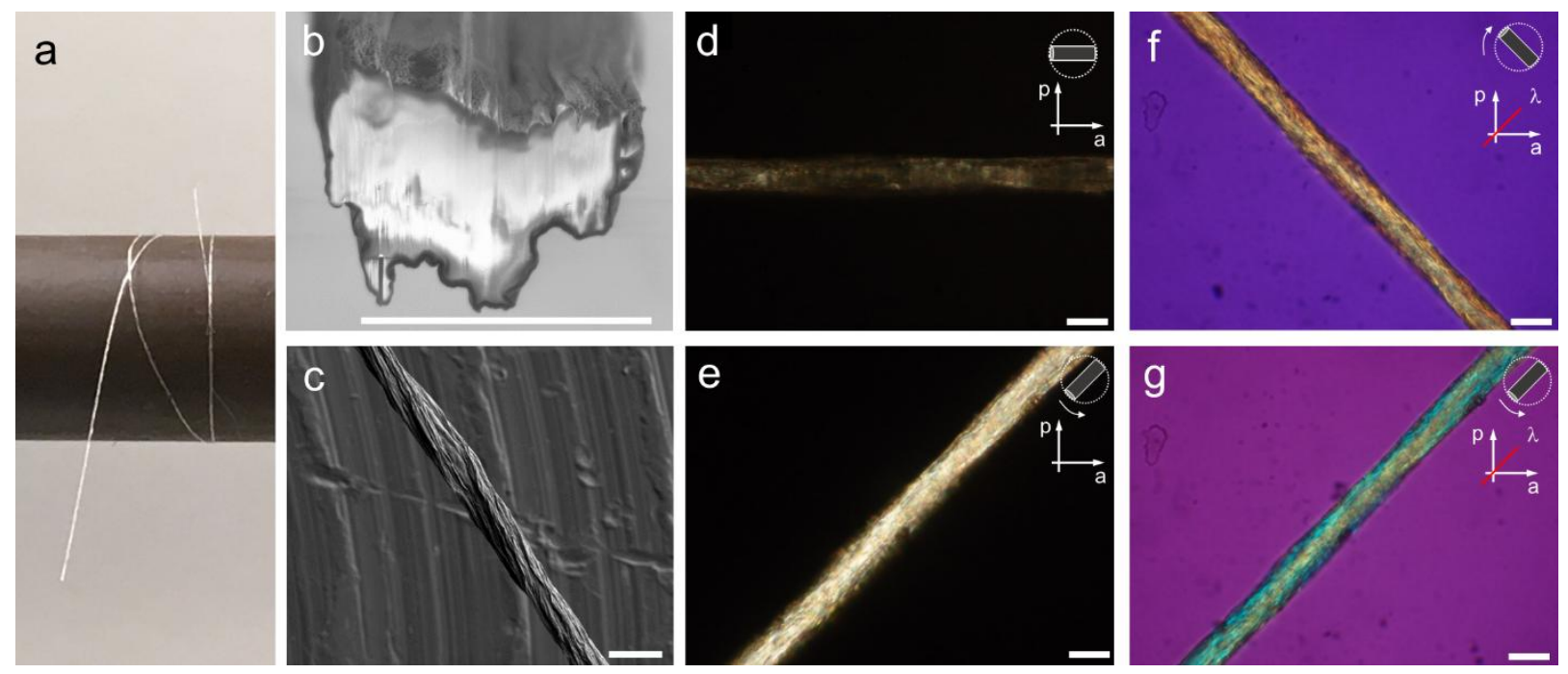

Figure 2. (a) Photograph of fiber section on spool, (b) SEM image of focused ion beam milled cross-section of Au-coated dry fiber, (c) SEM image of Au-coated dry fiber on Al stub, (d-g) Polarized optical microscopy images for fiber from INT-20d without (d, e) and with $(f, g)$ retardation plate $(\lambda$-plate, $530 \mathrm{~nm})$. The orientation of the polarizer and analyzer are indicated by labels $\mathrm{p}$ and a, respectively, while the red line represents the slow axis of the retardation plate $\lambda$. The fiber was tilted by $+/-45^{\circ}$ with respect to the horizontal direction as indicated in the upper right corner. Scale bars $20 \mu \mathrm{m}$.

Polarized optical microscopy (POM) observations indicate a strong birefringence in all fibers, with a maximum of transmission observed with the fiber axis at $45^{\circ}$ to the crossed 
polarizers (Figure 2d,e and S5). The use of a retardation plate of $530 \mathrm{~nm}$ gives rise to different interference colors depending on the orientation of the fiber relative to the slow axis of the $\lambda$-plate and shows that the nanotubes axes are aligned with the fiber axis (Figure $2 \mathrm{f}, \mathrm{g}$ ). X-ray scattering (XRS) patterns were used to quantify the orientation within the fibers (typical example in Figure 3a); in these pure fibers, all the observed scattering features can be attributed to the INTs. The intensity scattered by a single nanotube is located in reciprocal planes perpendicular to its long axis, at $Q_{z}=l \frac{2 \pi}{T}$, where $T$ is the period of the nanotube atomic structure along its long axis $(\mathrm{T} \approx 8.5 \AA)$ and $l$ is an integer. In these patterns the scattering features from the fibers are modulated angularly with the $l=0$ scattering signal located on the equator of the fiber scattering pattern and the (002) feature centered in the fiber direction, indicating the preferred orientation of the nanotubes along the fiber axis. ${ }^{28}$ The intensity on the $l=0$ plane (Figure $3 \mathrm{~b}$ and S7) exhibits oscillations characteristic of the squared form factor of DW Ge-INTs. ${ }^{32}$ Although the broad modulation around $2.6 \mathrm{~nm}^{-1}$ is flattened, the lack of sharp Bragg peaks indicates that any local structural organization is limited to bundles of a small size. ${ }^{43,44}$ The formation of larger, more ordered bundles is presumably hindered during the fast coagulation process, given the relatively large size and mass of the INTs ( 2-17 GDa), compared to typical polymer molecules which may crystallize. The angular distribution of the $l=0$ signals is used to quantitatively determine the orientation of nanotubes within the fibers. ${ }^{28,45,46}$ The angular distribution of scattering intensity in reciprocal space fits well to a Lorentzian distribution (Figure 3c and S8) which, when transformed to an orientation distribution function (ODF) in direct space, results in a Lorentzian function to the power $1.5{ }^{28}$ The Hermans order parameter, or $\left\langle P_{2}\right\rangle$, is used to characterise orientation between perfectly uniaxial $\left(\left\langle P_{2}\right\rangle=1\right)$ or randomly oriented $\left(\left\langle P_{2}\right\rangle=\right.$ $0)$; it was calculated from the direct space ODF as $\left\langle P_{2}\right\rangle=\frac{1}{2}\left(3\left\langle\cos ^{2} \phi\right\rangle-1\right)$. Calculated values range from 0.49 to 0.74 for the eight fiber samples studied by XRS (Table S1). 
Measurements performed at varying positions along the length of a single fiber section indicates that the degree of alignment is constant within each section, and the differences in orientation between samples are likely to arise from the manual handling of each fiber section.
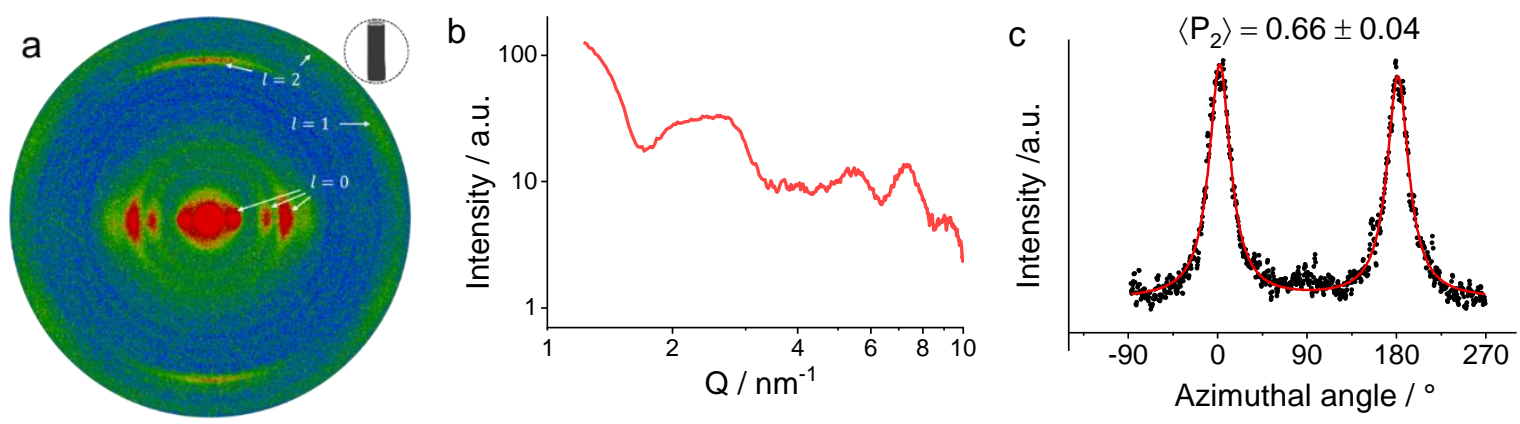

Figure 3. (a) False color X-ray scattering pattern of dry fiber from INT-20d, (b) Radial profile of scattered intensity in a sector $\pm 30^{\circ}$ from the equatorial plane after geometric and polarization corrections, (c) Azimuthal profile of scattered intensity at $2.6 \mathrm{~nm}^{-1}$ with Lorentzian fit and value of $\left\langle P_{2}\right\rangle$ calculated from direct space ODF

\section{MECHANICAL PROPERTIES OF DW Ge-INT FIBERS}

The mechanical properties of the pure INT fibers were measured using a conventional single filament tensile test. The tensile strength of dry fibers across the INT feedstocks follows the same trend observed in their gel-strengths, with a higher strength for feedstocks INT-12d to INT-40d and a lower strength for INT-5d. The data is quite scattered for these small batch fibers. However, within error, the strengths and stiffnesses of fibers produced from feedstocks INT-12d to INT-40d were similar with an ultimate tensile strength and elastic modulus in ambient conditions $(40 \% \mathrm{RH})$ of approximately $100 \mathrm{MPa}$ and $10 \mathrm{GPa}$ respectively (Figure 4a). The specific properties of the fibers can be determined using the breaking force $(10-50 \mathrm{mN})$ and estimated linear density $(\sim 0.4$ tex $)$ with corresponding tenacity of $0.02-0.12 \mathrm{~N} \mathrm{tex}^{-1}$. A lower failure stress was measured for the shorter INT-5d sample $(\sim 60 \mathrm{MPa})$. These values are similar to other networks of hydrophilic nanoscale 
elements such as cellulose nanofibers (tensile strength $\sim 10-500 \mathrm{MPa}$, elastic modulus $\sim 2$ $30 \mathrm{GPa}^{30,47}$ ), and to carbon nanotube fibers assembled from surfactant dispersions, ${ }^{48}$ but lower than highly optimized, denser carbon nanotube fibers spun from liquid crystalline mesophases which can have tensile strengths exceeding $4 \mathrm{GPa}$. In addition to the increased intrinsic strength and modulus, the significantly higher strengths of the optimized CNT fibers may be due to the much larger aspect ratio of the nanotubes. The best performing $\mathrm{CNTs}^{49}$ had an aspect ratio around 6700 , while the INTs in this work had an aspect ratio of $\sim 200$. An approximately linear relationship between fiber tensile strength and CNT aspect ratio has been reported previously, ${ }^{29}$ as might be expected below the critical stress transfer length in a discontinuous nanotube assembly. ${ }^{50}$ In this regime, the fibers fail by shear slip of nanotubes, which is illustrated by electron micrographs of fracture surfaces showing a 'finger-like' pull-out structure (Figure 4b). This fibrillar failure, due to nanotube slip, has previously been noticed in carbon nanotube fibers with a similar packing density., ${ }^{1,51}$ 

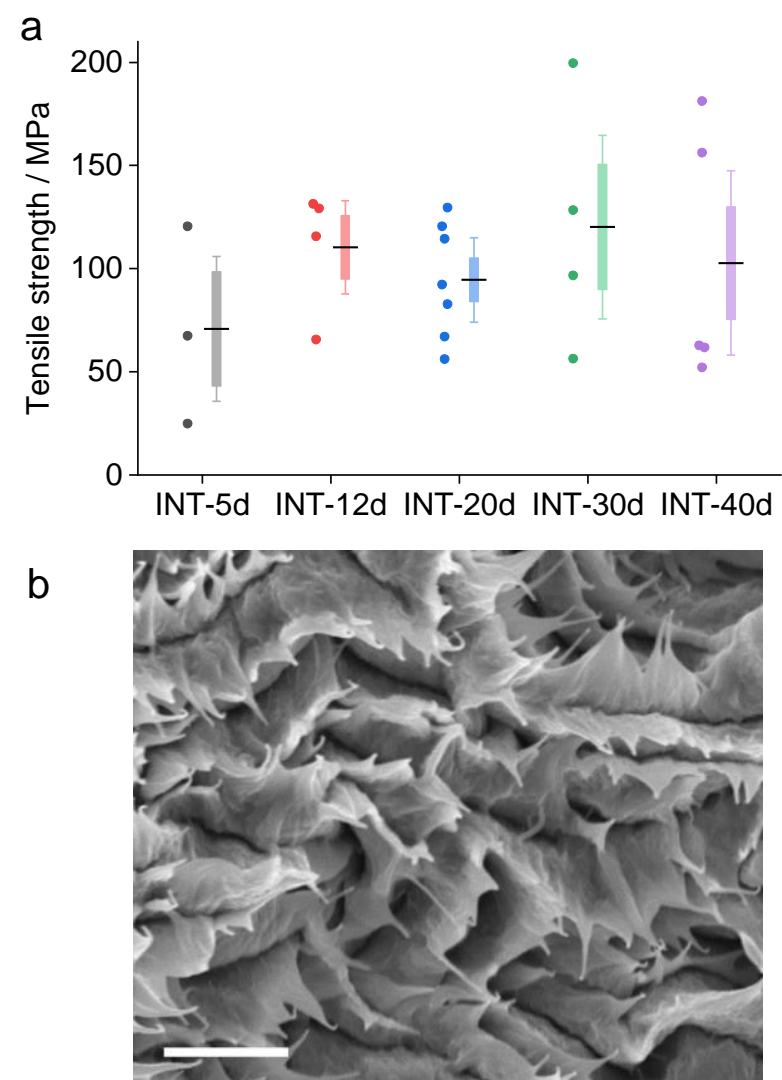

Figure 4. (a) Tensile strength of fibers for each feedstock, dots show individual test results, dashes show mean value, and box and whiskers show the standard error and $90 \%$ confidence interval for the mean, (b) SEM image of fracture surface of INT fiber showing pull-out failure. Scale bar $1 \mu \mathrm{m}$

The importance of shear interactions in determining the fiber mechanical properties is illustrated by the effect of humidity (Figure 5a). The modulus, in particular, is strongly dependent upon the humidity during the test, with a modulus of $30 \mathrm{GPa}$ at $10 \% \mathrm{RH}$ compared to $2.8 \mathrm{GPa}$ at $85 \% \mathrm{RH}$. Due to the strongly hydrophilic nature of both the INTs and the remnant $\mathrm{CaCl}_{2}$, increasing humidity leads to significant adsorption of water, with uptake reaching $150 \%$ by mass at $90 \% \mathrm{RH}$ (Figure $5 \mathrm{~b}$ and S6). The presence of water is expected to modify the interactions between the surface hydroxyl groups, leading to a reduction of both the shear strength and shear modulus of the inter-nanotube interactions, without affecting the intrinsic INT axial properties. The tenfold decrease in tensile strength and modulus with 
increasing humidity, therefore, indicates that the fiber properties are driven by inter-nanotube interactions, which are weakened by incorporation of water into the fiber structure. The change in failure mode of these INT fibers with relative humidity is clearly shown by the fracture surfaces (Figure 5c-e). Fibers tested at low relative humidity show a flat brittle fracture surface. As humidity is increased more pull-out is evident and at high relative humidity local necking and shear deformation is visible. The humidity dependence is broadly reversible, with fibers conditioned at $85 \% \mathrm{RH}$ and then tested at $40 \% \mathrm{RH}$ showing similar tensile behaviour to those simply conditioned at $40 \%$ (Figure S9).

Figure 5. (a) Stress-strain curves for fiber samples of INT-30d at controlled humidity, (b) Dynamic vapor sorption isotherms at $25^{\circ} \mathrm{C}$ showing water uptake with relative humidity for adsorption and desorption cycles, (c-e) optical micrographs of INT-30d fibers fractured at (c) $10 \%$, (d) $40 \%$ and (e) $85 \%$ relative humidity. Scale bars $20 \mu \mathrm{m}$.

In order to explore the scatter in fiber properties in more detail, a larger number of tensile samples were tested for INT-20d. The stress-strain curves exhibit the typical shape seen in nanomaterial fibers. There is a short initial take-up related to the straightening of fiber followed by an elastic region and then plastic deformation characteristic of inter-nanotube
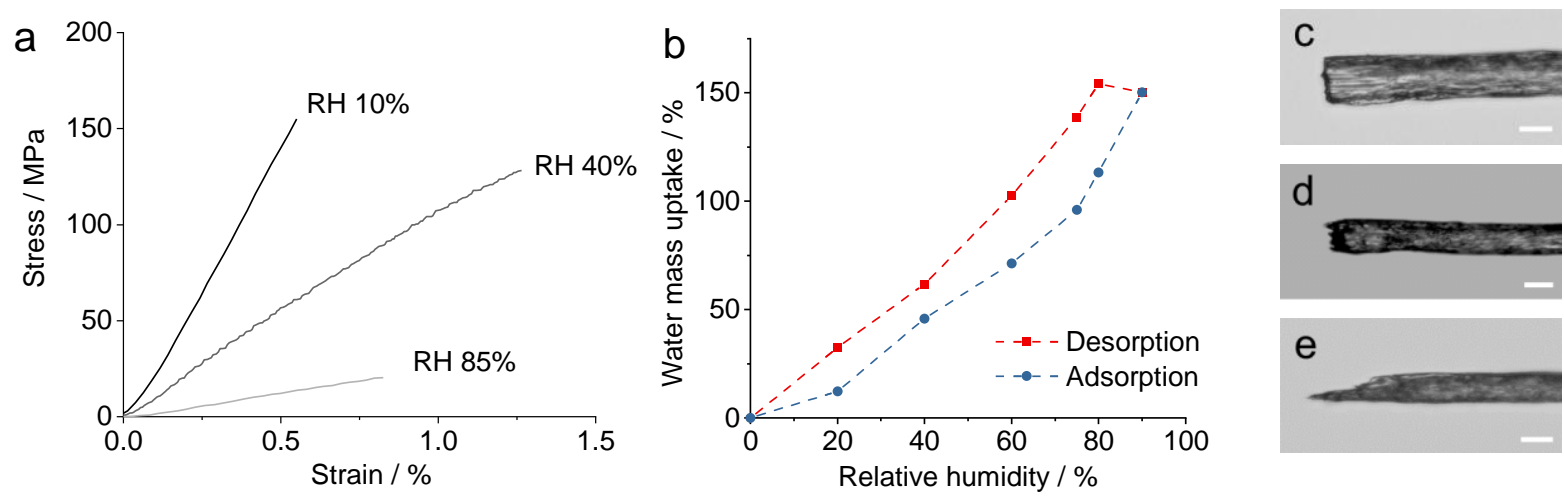
slip, ${ }^{52}$ indicated by a prominent 'elbow' at around $25 \mathrm{MPa}$ in most cases (Figure 6a). Perhaps surprisingly, higher degrees of alignment correlate strongly with lower elastic modulus and a higher strain-to-failure (Figure $6 \mathrm{~b}, \mathrm{c}$ ). This result initially seems to be counter-intuitive, 
particularly when considering typical short-fiber composite models which predict increased stiffness and strength with increased alignment. ${ }^{53,54}$
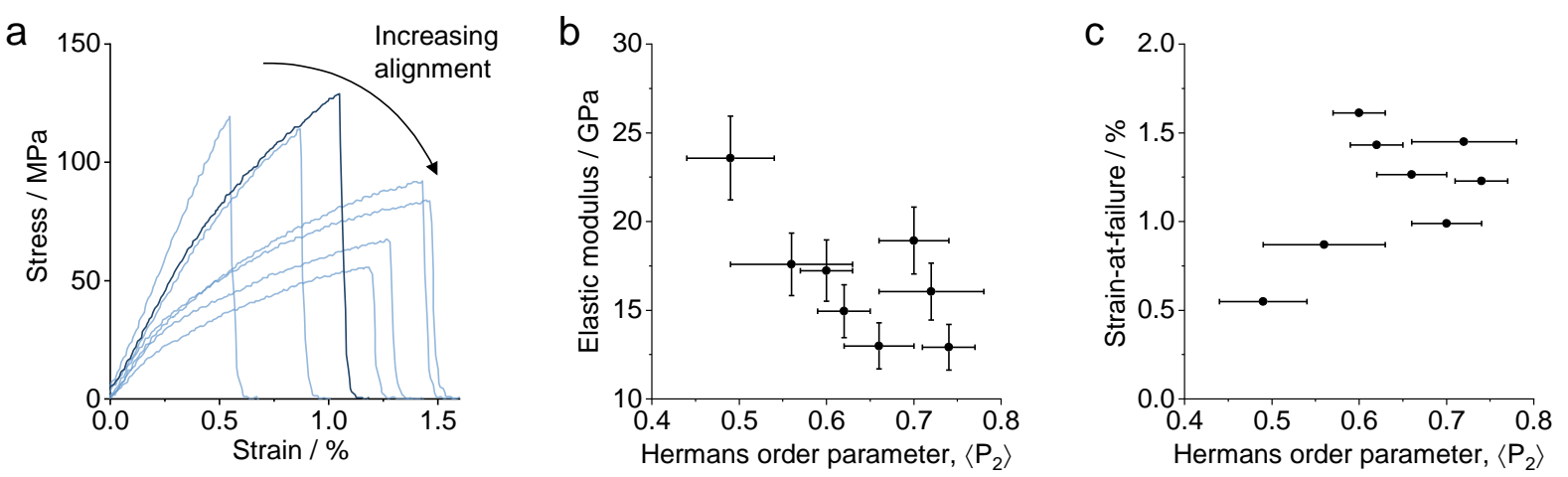

Figure 6. (a) Stress-strain curves of fiber samples from INT-20d, (b) elastic modulus and

(c) strain-at-failure as a function of Hermans order parameter

However, this trend may be explained by considering the microstructure and load transfer within these relatively porous fibers, in the absence of any matrix. The fibers are comprised of stiff, straight rods which have a low interfacial shear strength. In highly aligned fibers, load is transferred between the nanotubes by relatively mobile point contacts, which can slide without arrest, leading to ductile behaviour and a higher strain-to-failure. However, in less aligned packings of high aspect ratio rods, load can also be supported by mechanical interlocking which occurs through a jamming transition when the number of independent contacts per rod exceeds a critical value of around ten. ${ }^{55,56}$ This mechanism of load transfer has been observed in systems across a variety of length scales including bird nests, bamboo skewers and colloidal gels. ${ }^{57-59}$ As the number of contacts between rigid rods increases with increased misorientation, ${ }^{60}$ it is expected that jamming occurs more frequently in the less aligned INT fibers and leads to more efficient stress transfer within the fiber and hence a higher modulus. Deformation of these fibers may be considered as analogous to the shear of granular assemblies of frictional rigid rods. Such assemblies are modelled to undergo extensive shear alignment in order to reduce inter-particle contact and reduce the stress required for deformation. ${ }^{61}$ As the solid volume fraction of these models increases above 
$50 \%$, a rapid increase in shear stress occurs due to mechanical percolation through the formation of rigid clusters. We propose that the INT fibres are in this mechanical percolation regime, with a percolation threshold that increases with alignment. ${ }^{62}$ At a constant volume fraction, within this regime, less oriented systems are more networked. The granular modelling also highlights the importance of friction in determining the extent of network formation, which here correlates with the effects of humidity on mechanical response.

The orientational dependence is more manifest in these INT fibers than in analogous CNTs or nanocellulose fibers, due to the comparably short aspect ratio and high rigidity of the INTs. The individual INTs are less able to bend and form the necessary contacts for frictional load transfer, and do not kink or fibrillate in the manner of CNT and nanocellulose bundles. The coagulation / gelation process delivers porous fibres near the percolation threshold, and as a result, the jamming behaviour is most important to the fiber mechanical properties. At higher packing fractions, or with a composite matrix, ${ }^{28}$ a more conventional increase in mechanical properties with alignment can be anticipated.

\section{CONCLUSIONS}

Continuous macroscopic fibers of pure imogolite nanotubes can be prepared by lab scale wet spinning which, as a form of conventional coagulation spinning, can be readily optimized and scaled-up. In the future, these binder free fibers can be converted into more complex macroscale constructs by weaving, braiding, or other textile processes. They may find applications in catalysis or molecular filtration by providing a robust structure that enables reagent access to the monodisperse nanotubes and their nanoscale pores. In addition, once optimised, INT fibers may be used as reinforcements in structural composites. Whilst the absolute performance will be lower than carbon nanotube-based fibres, the improved surface interaction with the matrix, oxidation stability and optical transparency may offer advantages, just as glass fibres are often used in preference to carbon fibres in many composite 
applications. The ease of fiber spinning and the resulting fiber properties are both strongly linked to the length distribution of the INT feedstocks. Short INTs, less than $100 \mathrm{~nm}$, were challenging to spin, due to the low gel strength of the proto-fibers. However, longer INTs formed more robust gels, as well as stronger and stiffer dried fibers. The formation of a liquid crystal mesophase in the spinning dope likely contributes to the alignment of the INTs due to shear during spinning. However, in contrast to typical models of nanotube yarns, fiber strengths and stiffnesses were lower for more aligned fibers. This unusual finding can be attributed to the relatively low aspect ratio of the INTs and their high rigidity, which makes them much straighter than equivalent CNTs or biological nanofibrils, and less able to bend to form contacts. They are, therefore, much less likely to entangle or interlock in aligned fibers. Combined with relatively low strength inter-tube interactions, which are easily disrupted by ambient water, misorientation is needed to transfer load across the individual INTs within the porous fiber. Ductile failure occurs due first to frictional sliding of the INTs, followed by pull-out. The most significant factor currently affecting the mechanical properties of these INTs fibers is the relative humidity during tensile testing. Reducing the humidity removes the water from the inter-tube space, suppressing the plasticity associated with INT sliding at elevated humidity. As a result, the tensile strength and modulus of the fibers increase dramatically from 20 to $155 \mathrm{MPa}$ and 2.7 to $30 \mathrm{GPa}$, respectively, when reducing humidity from 85 to $10 \% \mathrm{RH}$. This result highlights the importance of controlling shear interactions in nanomaterial fibers, in general. The INT fibers provide a useful model system in which the shear interactions can be systematically explored through humidity. Through this system, an optimized relationship between aspect ratio, alignment, and interfacial shear properties may be identified which can be matched with other material systems. With further optimization of feedstock and spinning process, denser, stronger INT fibers may usefully complement CNT fibers in structural composites. 


\section{METHODS}

\section{Synthesis of DW Ge-INTs}

Double-walled Ge-INTs were synthesized using aluminum perchlorate nonahydrate (Reagent grade, Alfa Aesar), tetraethoxygermane (TEOG, $\geq 99.95 \%$, Sigma Aldrich) and urea ( $>99 \%$, Sigma Aldrich) following the procedure described elsewhere. ${ }^{32}$ TEOG was mixed in a PFTE beaker with an aqueous solution of aluminum perchlorate $\left(\mathrm{C}=0.2 \mathrm{~mol} \cdot \mathrm{L}^{-1}\right)$ and a urea solution with a molar ratio $[\mathrm{Ge}]:[\mathrm{Al}]:[\mathrm{urea}]=1: 2: 2$. After mixing, the PFTE beaker was placed in an acid digestion bomb (Zeoclave, Maximator, France) for hydrothermal treatment at $140^{\circ} \mathrm{C}$. The solution was recovered after 5 days and then dialyzed against ultrapure water using semi-permeable membranes (Spectra/Por ${ }^{\circledR}$, cut-off $\left.=10 \mathrm{kDa}\right)$ until the conductivity of bath drops below $0.5 \mathrm{mS} \mathrm{m}^{-1}$. $^{37}$

\section{Preparation of INT fibers}

INT fibers were prepared by injecting each INT feedstock through a 21 gauge needle at $5 \mathrm{~mL} / \mathrm{hr}$ (linear velocity $6.7 \mathrm{~mm} \mathrm{~s}^{-1}$ ) into a coagulation bath of aqueous calcium chloride $\left(300 \mathrm{~g} \mathrm{~L}^{-1}\right)$. The fibers were collected on a rotating PTFE wheel (diameter $100 \mathrm{~mm}$, surface linear velocity $33.5 \mathrm{~mm} \mathrm{~s}^{-1}$ ) with a spin draw ratio of 5 . After collection on the wheel, the fibers were cut into $15 \mathrm{~cm}$ lengths and washed by dipping in deionized water ( 3 x 2 seconds). A small tag of aluminium foil ( $20 \mathrm{mg})$ was attached to one end of each fiber and they were hung to dry in ambient conditions.

\section{Tensile testing of INT fibers}

INT fiber samples were tested following the standard BS ISO 11566:1996. Fiber samples were mounted onto card frames with a gauge length of $15 \mathrm{~mm}$ with the ends fixed with epoxy adhesive (Araldite Rapid, Huntsman Advanced Materials Ltd., GB). The tensile tests were conducted on a TST350 tensile stress tester (Linkam Scientific Instruments Ltd., GB) with a 
$2 \mathrm{~N}$ load cell and a crosshead speed of $1 \mathrm{~mm} \mathrm{~min}^{-1}$. The cross-sectional area for each sample was determined using the observed diameter in transmission optical microscopy.

\section{Characterization of INT fibers}

A small portion of INT feedstock was sampled and dried for further infrared (IR) characterization between $\mathrm{KBr}$ pressed pellets ( 1 wt $\%$ of dry DW Ge-INTs powder). IR spectra were acquired in transmission mode (Nicolet iS50), by averaging 256 scans at a resolution of $4 \mathrm{~cm}^{-1}$. Transmission electron microscopy (TEM) was performed on highly dilute dispersions of feedstock $\left(1 \mathrm{mg} \mathrm{L}^{-1}\right)$ prepared in ethanol and deposited in carbon-coated copper grid. TEM micrographs were recorded with a JEOL 1400 operating at $80 \mathrm{kV}$. X-ray scattering (XRS) experiments were carried out on a rotating anode (Model RU H3R, Rigaku Corporation, JP) using $\mathrm{CuK} \alpha$ radiation $(\lambda=0.154 \mathrm{~nm})$ delivered by a multilayer W/Si optics. Pieces of single fibers were mounted on cardboard struts and placed in the beam path. Some samples were imaged in lab atmosphere (for which a background due to scattering from air was subtracted) and others imaged using a vacuum chamber equipped with a collimator, with entrance and exit windows made of $500 \mathrm{~mm}$-thick Mylar film. The fiber was kept perpendicular to the incident X-ray beam. Two-dimensional patterns were recorded on a MAR345 detector (marXperts GmbH, DE) with $150 \mu \mathrm{m}$ pixel size. Extraction of the scattered intensity $I$ as a function of the scattering modulus $Q(Q=4 \pi \sin \theta=\lambda$ where $2 \theta$ is the scattering angle) or azimuthal angle $\tau$ were performed with home-developed software. Scanning electron microscopy (SEM) and energy dispersive x-ray spectroscopy (EDX) were carried out using a JEOL 6010LA microscope on $\mathrm{Au}$ sputter-coated samples at an accelerating voltage of $20 \mathrm{kV}$. Cross-sections were imaged using a Zeiss Auriga Crossbeam focused ion beam scanning electron microscope (FIB-SEM) after milling with a $30 \mathrm{kV} \mathrm{Ga}+$ ion beam at a beam current of $30 \mathrm{nA}$. The water sorption behavior of INT fibres was investigated through the Dynamic Vapour Sorption (DVS) technique using DVS 
Advantage apparatus (Surface Measurement Systems, UK). Several strands of INT fibre ( 13 mg) were manually shaped into a ball of sample and loaded in an aluminium pan. The moisture sorption was analyzed over a range of pre-set $\mathrm{RH}$ conditions starting from $0 \% \mathrm{RH}$ and increasing to $90 \% \mathrm{RH}$ in different $\mathrm{RH}$ steps at $25^{\circ} \mathrm{C}$, before decreasing to $0 \% \mathrm{RH}$ to obtain the desorption data. The moisture sorption measurements were recorded using the DVS Analysis Suite software.

\section{ASSOCIATED CONTENT}

Supporting information. Infrared spectra of DW Ge-INTs; crossed polarizer optical observations of aqueous DW Ge-INT suspensions; schematic of modified Wilhelmy method; SEM images of focused ion beam milled cross-sections and energy dispersive spectroscopy of INT fiber; crossed polarizer optical images of INT fibers; dynamic vapor sorption isotherm of DW Ge-INTs; radial profiles of scattered X-ray intensity; azimuthal profiles of scattered X-ray intensity; stress-strain curves of fibers tested at $40 \% \mathrm{RH}$ with and without initial conditioning at $85 \% \mathrm{RH}$; table of structural parameters and mechanical properties; note on calculation of packing density. This material is available free of charge via the Internet at http://pubs.acs.org.

\section{AUTHOR INFORMATION}

\section{Corresponding Author}

*pascale.launois@universite-paris-saclay.fr

*m.shaffer@imperial.ac.uk

\section{Author Contributions}

The manuscript was written through contributions of all authors. All authors have given approval to the final version of the manuscript. 


\section{ACKNOWLEDGMENTS}

The authors would like to thank DSTL for the funding received for this project through the UK-France PhD Scheme. The authors would like to acknowledge use of characterization facilities within the Harvey Flower Electron Microscopy Suite, Department of Materials, Imperial College London. The present work has benefited from Imagerie-Gif core facility supported by l'Agence Nationale de la Recherche (ANR-11-EQPX-0029/Morphoscope; ANR-10-INBS-04/FranceBioImaging; ANR-11-IDEX-0003-02/Saclay Plant Sciences). We are grateful to C. Goldmann for her support with TEM observations. X-ray scattering experiments were performed on the MORPHEUS platform of experiments at Laboratoire de Physique des Solides. We thank S. Rouzière and P.-A. Albouy for their support with the Xray scattering experiments and V. Karde and J. Heng for conducting the DVS measurement. 


\section{REFERENCES}

1. Vilatela, J. J.; Windle, A. H., Yarn-Like Carbon Nanotube Fibers. Advanced Materials 2010, 22, 4959-4963.

2. Koziol, K.; Vilatela, J.; Moisala, A.; Motta, M.; Cunniff, P.; Sennett, M.; Windle, A., High-Performance Carbon Nanotube Fiber. Science 2007, 318, 1892-1895.

3. Behabtu, N.; Young, C. C.; Tsentalovich, D. E.; Kleinerman, O.; Wang, X.; Ma, A. W. K.; Bengio, E. A.; ter Waarbeek, R. F.; de Jong, J. J.; Hoogerwerf, R. E.; Fairchild, S. B.; Ferguson, J. B.; Maruyama, B.; Kono, J.; Talmon, Y.; Cohen, Y.; Otto, M. J.; Pasquali, M., Strong, Light, Multifunctional Fibers of Carbon Nanotubes with Ultrahigh Conductivity. Science 2013, 339, 182-186.

4. Jeong, H. D.; Kim, S. G.; Choi, G. M.; Park, M.; Ku, B.-C.; Lee, H. S., Theoretical and Experimental Investigation of the Wet-Spinning Process for Mechanically Strong Carbon Nanotube Fibers. Chemical Engineering Journal 2021, 412, 128650.

5. Mikhalchan, A.; Vilatela, J. J., A Perspective on High-Performance CNT Fibres for Structural Composites. Carbon 2019, 150, 191-215.

6. Paineau, E., Imogolite Nanotubes: A Flexible Nanoplatform with Multipurpose Applications. Applied Sciences 2018, 8, 1921.

7. Cradwick, P. D. G.; Farmer, V. C.; Russell, J. D.; Masson, C. R.; Wada, K.; Yoshinaga, N., Imogolite, a Hydrated Aluminium Silicate of Tubular Structure. Nature Physical Science 1972, 240 (104), 187-189.

8. Paineau, E.; Launois, P., Nanomaterials from Imogolite: Structure, Properties and Functional Applications. Elsevier: 2019; pp 257-284. 
9. Konduri, S.; Mukherjee, S.; Nair, S., Controlling Nanotube Dimensions: Correlation between Composition, Diameter, and Internal Energy of Single-Walled Mixed Oxide Nanotubes. ACS Nano 2007, 1 (5), 393-402.

10. Maillet, P.; Levard, C.; Larquet, E.; Mariet, C.; Spalla, O.; Menguy, N.; Masion, A.; Doelsch, E.; Rose, J.; Thill, A., Evidence of Double-Walled Al-Ge Imogolite-Like Nanotubes. A Cryo-TEM and SAXS Investigation. Journal of the American Chemical Society 2010, 132 (4), 1208-1209.

11. Thill, A.; Maillet, P.; Guiose, B.; Spalla, O.; Belloni, L.; Chaurand, P.; Auffan, M.; Olivi, L.; Rose, J., Physico-Chemical Control over the Single- or Double-Wall Structure of Aluminogermanate Imogolite-Like Nanotubes. J Am Chem Soc 2012, 134 (8), 3780-6.

12. Jiang, C.; Saha, A.; Xiang, C.; Young, C. C.; Tour, J. M.; Pasquali, M.; Martí, A. A., Increased Solubility, Liquid-Crystalline Phase, and Selective Functionalization of SingleWalled Carbon Nanotube Polyelectrolyte Dispersions. ACS Nano 2013, 7, 4503-4510.

13. Davis, V. A.; Parra-Vasquez, A. N. G.; Green, M. J.; Rai, P. K.; Behabtu, N.; Prieto, V.; Booker, R. D.; Schmidt, J.; Kesselman, E.; Zhou, W.; Fan, H.; Adams, W. W.; Hauge, R. H.; Fischer, J. E.; Cohen, Y.; Talmon, Y.; Smalley, R. E.; Pasquali, M., True Solutions of Single-Walled Carbon Nanotubes for Assembly into Macroscopic Materials. Nature Nanotechnology 2009, 4, 830-834.

14. Kleinerman, O.; Liberman, L.; Behabtu, N.; Pasquali, M.; Cohen, Y.; Talmon, Y., Direct Imaging of Carbon Nanotube Liquid-Crystalline Phase Development in True Solutions. Langmuir 2017, 33, 4011-4018. 
15. Paineau, E.; Krapf, M. E.; Amara, M. S.; Matskova, N. V.; Dozov, I.; Rouziere, S.; Thill, A.; Launois, P.; Davidson, P., A Liquid-Crystalline Hexagonal Columnar Phase in Highly-Dilute Suspensions of Imogolite Nanotubes. Nat Commun 2016, 7, 10271.

16. Paineau, E.; Rouziere, S.; Monet, G.; Diogo, C. C.; Morfin, I.; Launois, P., Role of Initial Precursors on the Liquid-Crystalline Phase Behavior of Synthetic Aluminogermanate Imogolite Nanotubes. J Colloid Interface Sci 2020, 580, 275-285.

17. Ohashi, F.; Tomura, S.; Akaku, K.; Hayashi, S.; Wada, S. I., Characterization of Synthetic Imogolite Nanotubes as Gas Storage. Journal of Materials Science 2004, 39 (5), 1799-1801.

18. Liao, Y.-Y.; Picot, P.; Brubach, J.-B.; Roy, P.; Thill, A.; Le Caër, S., Water Adsorption in Single- and Double-Walled Inorganic Nanotubes. The Journal of Physical Chemistry C 2019, 123 (32), 19768-19777.

19. Li, L.; Ma, W.; Takada, A.; Takayama, N.; Takahara, A., Organic-Inorganic Hybrid Films Fabricated from Cellulose Fibers and Imogolite Nanotubes. Biomacromolecules 2019, 20 (9), 3566-3574.

20. Benitez, A. J.; Torres-Rendon, J.; Poutanen, M.; Walther, A., Humidity and Multiscale Structure Govern Mechanical Properties and Deformation Modes in Films of Native Cellulose Nanofibrils. Biomacromolecules 2013, 14 (12), 4497-506.

21. González, R. I.; Rogan, J.; Bringa, E. M.; Valdivia, J. A., Mechanical Response of Aluminosilicate Nanotubes under Compression. The Journal of Physical Chemistry C 2016, 120 (26), 14428-14434. 
22. da Silva, M. C.; dos Santos, E. C.; Lourenço, M. P.; Gouvea, M. P.; Duarte, H. A., Structural, Electronic, and Mechanical Properties of Inner Surface Modified Imogolite Nanotubes. Frontiers in Materials 2015, 2.

23. Lourenço, M. P.; Guimarães, L.; da Silva, M. C.; de Oliveira, C.; Heine, T.; Duarte, H. A., Nanotubes with Well-Defined Structure: Single- and Double-Walled Imogolites. The Journal of Physical Chemistry C 2014, 118 (11), 5945-5953.

24. Liu, C.-H.; Kang, D.-Y., Influence of Interwall Interaction in Double-Walled Aluminogermanate Nanotubes on Mechanical Properties. Computational Materials Science 2017, 135, 54-63.

25. Kang, D.-Y.; Lydon, M. E.; Yucelen, G. I.; Jones, C. W.; Nair, S., SolutionProcessed Ultrathin Aluminosilicate Nanotube-Poly(Vinyl Alcohol) Composite Membranes with Partial Alignment of Nanotubes. ChemNanoMat 2015, 1 (2), 102-108.

26. Kang, D. Y.; Tong, H. M.; Zang, J.; Choudhury, R. P.; Sholl, D. S.; Beckham, H. W.; Jones, C. W.; Nair, S., Single-Walled Aluminosilicate Nanotube/Poly(Vinyl Alcohol) Nanocomposite Membranes. ACS Appl Mater Interfaces 2012, 4 (2), 965-76.

27. Ma, W.; Yah, W. O.; Otsuka, H.; Takahara, A., Application of Imogolite Clay Nanotubes in Organic-Inorganic Nanohybrid Materials. Journal of Materials Chemistry 2012, 22 (24).

28. Lee, W. J.; Paineau, E.; Anthony, D. B.; Gao, Y.; Leese, H. S.; Rouziere, S.; Launois, P.; Shaffer, M. S. P., Inorganic Nanotube Mesophases Enable Strong Self-Healing Fibers. ACS Nano 2020, 14 (5), 5570-5580. 
29. Tsentalovich, D. E.; Headrick, R. J.; Mirri, F.; Hao, J.; Behabtu, N.; Young, C. C.; Pasquali, M., Influence of Carbon Nanotube Characteristics on Macroscopic Fiber Properties. ACS Applied Materials \& Interfaces 2017, 9, 36189-36198.

30. Clemons, C., Nanocellulose in Spun Continuous Fibers: A Review and Future Outlook. Journal of Renewable Materials 2016, 4 (5), 327-339.

31. Farmer, V. C.; Fraser, A. R.; Tait, J. M., Synthesis of Imogolite: A Tubular Aluminium Silicate Polymer. Journal of the Chemical Society, Chemical Communications 1977, (13), 462-463.

32. Amara, M. S.; Paineau, E.; Bacia-Verloop, M.; Krapf, M. E.; Davidson, P.; Belloni, L.; Levard, C.; Rose, J.; Launois, P.; Thill, A., Single-Step Formation of Micron Long $(\mathrm{OH})_{3} \mathrm{Al}_{2} \mathrm{O}_{3} \mathrm{Ge}(\mathrm{OH})$ Imogolite-Like Nanotubes. Chem Commun (Camb) 2013, 49 (96), 11284-6.

33. Du, P.; Yuan, P.; Thill, A.; Annabi-Bergaya, F.; Liu, D.; Wang, S., Insights into the Formation Mechanism of Imogolite from a Full-Range Observation of its Sol-Gel Growth. Applied Clay Science 2017, 150, 115-124.

34. Yang, H.; Wang, C.; Su, Z., Growth Mechanism of Synthetic Imogolite Nanotubes. Chemistry of Materials 2008, 20 (13), 4484-4488.

35. Yucelen, G. I.; Kang, D.-Y.; Schmidt-Krey, I.; Beckham, H. W.; Nair, S., A Generalized Kinetic Model for the Formation and Growth of Single-Walled Metal Oxide Nanotubes. Chemical Engineering Science 2013, 90, 200-212.

36. Maillet, P.; Levard, C.; Spalla, O.; Masion, A.; Rose, J.; Thill, A., Growth Kinetic of Single and Double-Walled Aluminogermanate Imogolite-Like Nanotubes: An Experimental and Modeling Approach. Phys Chem Chem Phys 2011, 13 (7), 2682-9. 
37. Paineau, E.; Monet, G.; Peyre, V.; Goldmann, C.; Rouziere, S.; Launois, P., Colloidal Stability of Imogolite Nanotube Dispersions: A Phase Diagram Study. Langmuir 2019, 35 (38), 12451-12459.

38. Chang, C.; Zhao, Y.; Liu, Y.; An, L., Liquid Crystallinity of Carbon Nanotubes. RSC Advances 2018, 8 (28), 15780-15795.

39. Shikinaka, K.; Koizumi, Y.; Kaneda, K.; Osada, Y.; Masunaga, H.; Shigehara, K., Strain-Induced Reversible Isotropic-Anisotropic Structural Transition of Imogolite Hydrogels. Polymer 2013, 54 (10), 2489-2492.

40. Park, K.-L.; Ma, W.; Higaki, Y.; Takahara, A., Design and Characterization of Hybrid Hydrogels Composed of Imogolite Fibrous Nanotubular Clay and Hyaluronic Acid. Polymer 2016, 100, 238-243.

41. Jiang, C.; Saha, A.; Young, C. C.; Hashim, D. P.; Ramirez, C. E.; Ajayan, P. M.; Pasquali, M.; Martí, A. A., Macroscopic Nanotube Fibers Spun from Single-Walled Carbon Nanotube Polyelectrolytes. ACS Nano 2014, 8, 9107-9112.

42. Khoshnevis, H.; Tran, T. Q.; Mint, S. M.; Zadhoush, A.; Duong, H. M.; Youssefi, M., Effect of Alignment and Packing Density on the Stress Relaxation Process of Carbon Nanotube Fibers Spun from Floating Catalyst Chemical Vapor Deposition Method. Colloids and Surfaces A: Physicochemical and Engineering Aspects 2018, 558, 570-578.

43. Amara, M. S.; Rouzière, S.; Paineau, E.; Bacia-Verloop, M.; Thill, A.; Launois, P., Hexagonalization of Aluminogermanate Imogolite Nanotubes Organized into Closed-Packed Bundles. The Journal of Physical Chemistry C 2014, 118 (17), 9299-9306. 
44. Paineau, E.; Amara, M. S.; Monet, G.; Peyre, V.; Rouzière, S.; Launois, P., Effect of Ionic Strength on the Bundling of Metal Oxide Imogolite Nanotubes. The Journal of Physical Chemistry C 2017, 121 (39), 21740-21749.

45. Pichot, V.; Badaire, S.; Albouy, P. A.; Zakri, C.; Poulin, P.; Launois, P., Structural and Mechanical Properties of Single-Wall Carbon Nanotube Fibers. Physical Review B 2006, $74(24)$.

46. Alemán, B.; Reguero, V.; Mas, B.; Vilatela, J. J., Strong Carbon Nanotube Fibers by Drawing Inspiration from Polymer Fiber Spinning. ACS Nano 2015, 9, 7392-7398.

47. Siró, I.; Plackett, D., Microfibrillated Cellulose and New Nanocomposite Materials: A Review. Cellulose 2010, 17 (3), 459-494.

48. Poulin, P.; Vigolo, B.; Launois, P., Films and Fibers of Oriented Single Wall Nanotubes. Carbon 2002, 40, 1741-1749.

49. Taylor, L. W.; Dewey, O. S.; Headrick, R. J.; Komatsu, N.; Peraca, N. M.; Wehmeyer, G.; Kono, J.; Pasquali, M., Improved Properties, Increased Production, and the Path to Broad Adoption of Carbon Nanotube Fibers. Carbon 2021, 171, 689-694.

50. Henry, J.; Pimenta, S., Semi-Analytical Simulation of Aligned Discontinuous Composites. Composites Science and Technology 2017, 144, 230-244.

51. Zhang, X.; Li, Q.; Holesinger, T. G.; Arendt, P. N.; Huang, J.; Kirven, P. D.; Clapp, T. G.; DePaula, R. F.; Liao, X.; Zhao, Y.; Zheng, L.; Peterson, D. E.; Zhu, Y., Ultrastrong, Stiff, and Lightweight Carbon-Nanotube Fibers. Advanced Materials 2007, 19, 4198-4201. 
52. Park, J.; Lee, J.; Lee, D.-M.; Lee, S.-H.; Jeong, H. S.; Lee, K.-H.; Kim, S. M., Mathematical Model for the Dynamic Mechanical Behavior of Carbon Nanotube Yarn in Analogy with Hierarchically Structured Bio-Materials. Carbon 2019, 152, 151-158.

53. Yoon, H. N., Strength of Fibers from Wholly Aromatic Polyesters. Colloid and Polymer Science 1990, 268 (3), 230-239.

54. Fernández-Toribio, J. C.; Alemán, B.; Ridruejo, Á.; Vilatela, J. J., Tensile Properties of Carbon Nanotube Fibres Described by the Fibrillar Crystallite Model. Carbon 2018, 133, 44-52.

55. Philipse, A. P., The Random Contact Equation and Its Implications for (Colloidal) Rods in Packings, Suspensions, and Anisotropic Powders. Langmuir 1996, 12 (5), 1127 1133.

56. Wouterse, A.; Luding, S.; Philipse, A. P., On Contact Numbers in Random Rod Packings. Granular Matter 2009, 11 (3), 169-177.

57. Weiner, N.; Bhosale, Y.; Gazzola, M.; King, H., Mechanics of Randomly Packed Filaments - the "Bird Nest" as Meta-Material. Journal of Applied Physics 2020, 127 (5).

58. Solomon, M. J.; Spicer, P. T., Microstructural Regimes of Colloidal Rod Suspensions, Gels, and Glasses. Soft Matter 2010, 6 (7), 1391-1400.

59. Blouwolff, J.; Fraden, S., The Coordination Number of Granular Cylinders. Europhysics Letters (EPL) 2006, 76 (6), 1095-1101.

60. Toll, S., Note: On the Tube Model for Fiber Suspensions. Journal of Rheology 1993, $37(1), 123-125$. 
61. Guo, Y.; Wassgren, C.; Hancock, B.; Ketterhagen, W.; Curtis, J., Granular Shear Flows of Flat Disks and Elongated Rods without and with Friction. Physics of Fluids 2013, $25(6)$.

62. Rahatekar, S. S.; Shaffer, M. S. P.; Elliott, J. A., Modelling Percolation in Fibre and Sphere Mixtures: Routes to More Efficient Network Formation. Composites Science and Technology 2010, 70 (2), 356-362. 
For Table of Contents Only

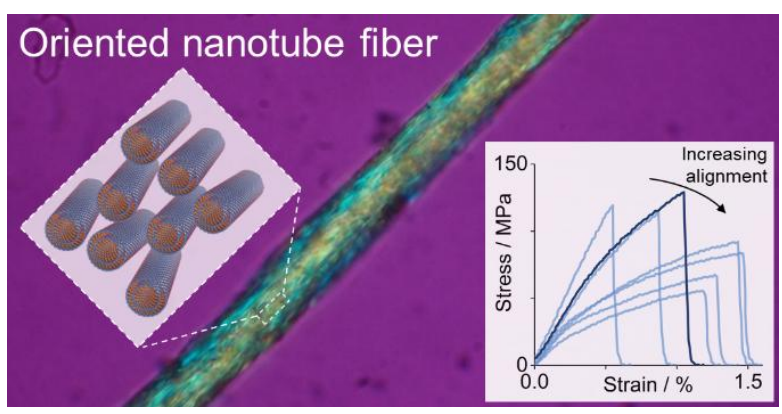

